\title{
EFFECT OF PEROXISOME PROLIFERATOR-ACTIVATED RECEPTOR-ALPHA-AGONISTS ON DIABETES-INDUCED ACUTE KIDNEY INJURY: ROLE OF OXIDATIVE STRESS AND HYPERLIPIDEMIA
}

\author{
VISHAL ARVIND CHAKKARWAR*, PRAVIN KAWTIKWAR \\ Department of Pharmacology, SN Institute of Pharmacy, Pusad, Maharashtra, India. Email: vishalcp1811@gmail.com \\ Received: 28 August 2018, Revised and Accepted: 10 September 2018
}

ABSTRACT

Objective: The present study investigated the possible effect of fenofibrate and gemfibrozil peroxisome proliferator-activated receptor-alpha agonist in diabetes-induced acute kidney injury (AKI) in rats.

Methods: Rats were administered streptozotocin (STZ) (50 mg/kg, i.p., single dose) to induce experimental diabetes mellitus. The development of diabetic AKI was assessed biochemically and histologically. In addition, the diabetes-induced lipid profile and renal oxidative stress were assessed. The single dose of STZ produced diabetes, which induced renal oxidative stress, altered the lipid profile and subsequently produced kidney injuryAKI in 7 weeks by increasing serum creatinine, blood urea nitrogen (BUN), proteinuria, and glomerular damage. Treatment with fenofibrate and gemfibrozil (30 mg/kg p.o, 7 weeks) normalized the altered lipid profile by decreasing serum cholesterol, triglycerides, and increasing serum highdensity lipoprotein in diabetic rats. Lisinopril (1 mg/kg, p.o., 7 weeks, reference compound) prevents lipid alteration and development of diabetic AKI.

Result: Fenofibrate and gemfibrozil, besides hyperglycemia, significantly prevented the development of diabetic AKI by reducing (serum and tissue) oxidative stress, hyperlipidemia, serum BUN, creatinine, and urinary protein. Further, fenofibrate, but not gemfibrozil, considerably reduced renal structural and functional abnormalities in diabetic rats. The fenofibrate was more effective in attenuating the diabetes-induced AKI and renal oxidative stress as compared to treatment with and gemfibrozil.

Conclusion: The fenofibrate and gemfibrozil treatment markedly prevented the diabetes-induced AKI. In comparison, the fenofibrate is found to be a superior approach to attenuate the diabetic AKI than gemfibrozil.

Keywords: Diabetes, Fenofibrate, Gemfibrozil, Nephropathy.

(C) 2019 The Authors. Published by Innovare Academic Sciences Pvt Ltd. This is an open access article under the CC BY license (http://creativecommons. org/licenses/by/4. 0/) DOI: http://dx.doi.org/10.22159/ajpcr.2019.v12i1.29397

\section{INTRODUCTION}

Diabetes is a metabolic disorder characterized by hyperglycemia followed by micro- and macro-vascular complications [1]. Diabetic nephropathy is an important microvascular complication of diabetes and is widely recognized as the most common cause of the endstage renal failure. Diabetes mediated acute kidney injury (AKI) is one of the leading causes of morbidity and mortality. Hyperglycemia, vascular endothelial dysfunction, and hyperlipidemia are considered as possible reasons behind the pathogenesis and progression of diabetic nephropathy [2]. Hyperglycemia mainly targets the almost all the kidney cellular elements such as glomerular endothelial cells, mesangial cells, podocytes, and tubular epithelial injury.

Further, the growing glomerulosclerosis, thickening of the glomerular basement membrane, glomerular hypertrophy, mesangial cell expansion, podocyte loss, renal-cell hypertrophy, and tubulointerstitial fibrosis are major pathological changes during the course of diabetic nephropathy, which ultimately results in functional consequences, including progressive albuminuria, reduction in glomerular filtration rate, elevation of arterial blood pressure, and fluid retention [3]. It is interesting that recent studies demonstrated numerous pleiotropic effects of peroxisome proliferator-activated receptor-alpha (PPAR- $\alpha$ ) agonist such as fenofibrate and gemfibrozil. Gemfibrozil is well known to produce antihyperlipidemic action by activation of PPAR- $\alpha$. In addition to this, upregulation of PPAR- $\alpha$ had an important role in affording the protection against experimental acute kidney failure. A recent study revealed the mechanism of cardioprotection by gemfibrozil by attenuating oxidative stress. Importantly, research exhibiting the most pronounced benefits of gemfibrozil in preventing vascular events by direct activation and protection of guanylyl cyclase (sGC) the key mediator of NO signaling [4]. The gemfibrozil mediated activation of guanylyl cyclase may be a rational concept to attenuate the oxidative stress and hyperlipidemia. Further, fenofibrate is well documented to attenuate diabetes-induced endothelial function by upregulating eNOS and by decreasing oxidative stress and hyperlipidemia [5]. Further, the nephroprotective role of fenofibrate is reported in diabetes by ameliorating oxidative stress and hyperlipidemia. Furthermore, fenofibrate has been shown to reduce glomerular hypertrophy, mesangial matrix expansion and suppress the expression of plasminogen activator inhibitor-1 and transforming growth factor-b $[5,6]$. Therefore, the renovascular protective role of PPAR- $\alpha$ agonist may be a rational therapeutic strategy to ameliorate diabetes mediated AKI.

\section{METHODS}

\section{Experimental animals}

The Institutional Animal Ethical Committee approved the experimental protocol used in the present study. Age-matched young Wistar rats weighing about 200-250 g were employed in the present study. The animals were housed in the room maintained at approximately $24 \pm 1^{\circ} \mathrm{C}$ temperature and humidity of $55 \pm 5 \%$ with 12 -h light/dark cycle. Free access to food (standard chow from Ashirwad Industries, Ropar, India) and water was allowed. The animals were acclimatized for at least 3-4 days before the initiation of the experiment and were observed for any sign of disease. The animals were maintained under proper conditions until the termination of the experiment. The animals were sacrificed after a predetermined period of the treatment as per the study design to evaluate various parameters. 
Experimental protocol

Seven groups were employed in the present study and each group comprised 8 animals each. The fenofibrate and gemfibrozil were dissolved in $1 \% \mathrm{w} / \mathrm{v}$ of carboxymethylcellulose (CMC) and water, respectively.

\section{Drug-treated diabetic group}

Group I (normal control)

Rats were maintained on standard food and water, and no treatment was given.

\section{Group II (diabetic control)}

Rats were administered streptozotocin (STZ) $(50 \mathrm{mg} / \mathrm{kg}$, i.p., once) dissolved in citrate buffer ( $\mathrm{pH} 4.5)$.

\section{Group III (fenofibrate per se)}

Rats were administered fenofibrate $(30 \mathrm{mg} / \mathrm{kg}$ p.o.) dissolved in $1 \% \mathrm{w} / \mathrm{v}$ CMC for 7 weeks.

\section{Group IV (gemfibrozil per se)}

Rats were administered gemfibrozil ( $30 \mathrm{mg} / \mathrm{kg}$ p.o.) dissolved in water for 7 weeks.

\section{Group V (fenofibrate treated diabetic group)}

The diabetic rats after 1 week of STZ administration were treated with fenofibrate (30 mg $/ \mathrm{kg}$ p.o.) for 7 weeks.

\section{Group VI (gemfibrozil treated diabetic group)}

The diabetic rats after 1 week of STZ administration were treated with gemfibrozil (30 mg/kg p.o.) for 7 weeks.

\section{Group VII (lisinopril treated diabetic group)}

The diabetic rats after 1 week of STZ administration were treated with lisinopril (1 mg/kg p.o.) for 7 weeks (Fig. 1).

\section{Drugs and chemicals}

STZ and acetylcholine hydrochloride were obtained from Sigma-Aldrich Ltd, St. Louis, USA. 1,1,3,3 tetra methoxypropane and carboxymethyl cellulose were purchased from V. K. Chemicals and Instruments, Ambala, India. The fenofibrate and gemfibrozil were obtained from Ranbaxy Laboratory Ltd., Gurgaon, India. Lisinopril was obtained from Dr. Reddy's Laboratory Ltd., Hyderabad, India. All other chemicals used in the present study were of analytical grade.

\section{Estimation of serum glucose}

At the end of the experimental protocol, the blood samples were collected, and serum was separated after allowing to clot followed by centrifugation. The glucose concentration was estimated by glucose oxidase-peroxidase GOD-POD method using the commercially available kit (Bio Lab Diagnostics India Private Limited) [5]

\section{Assessment of lipid profile}

Estimation of serum total cholesterol

The total cholesterol was estimated by cholesterol oxidase peroxidase CHOD-POD method using the commercially available kit (Bio Lab Diagnostics India Private Limited) [5,6].

\section{Estimation of serum triglycerides}

The serum triglyceride was estimated by GOD-POD method using the commercially available kit (Bio Lab Diagnostics India Private Limited) $[5,6]$

\section{Estimation of high-density lipoprotein (HDL)}

The HDL was estimated by CHOD-POD method using the commercially available kit (Bio Lab Diagnostics India Private Limited) [5,6].

\section{Assessment of oxidative stress}

The oxidative stress in serum samples was assessed by estimating serum thiobarbituric acid reactive substance (TBARS) and superoxide anion generation.

\section{Estimation of TBARS}

Serum

$1 \mathrm{ml}$ of $20 \%$ trichloroacetic acid was added to $100 \mu \mathrm{L}$ of serum and $1 \%$ TBA reagent $(1.0 \mathrm{~mL})$ which were mixed and incubated at $100^{\circ} \mathrm{C}$ for $30 \mathrm{~min}$. After cooling on ice, samples were centrifuged at $1000 \mathrm{~g}$ for $20 \mathrm{~min}$. Serum concentration of TBARS was measured spectrophotometrically at $532 \mathrm{~nm}$. A standard curve using 1, 1, 3, 3-tertramethoxyopropane $(1-10 \mu \mathrm{M})$ was plotted to calculate the concentration of TBARS $[5,6]$.

Tissue

The kidney was excised and washed with ice-cold isotonic saline and weighed. The kidney weight to body weight ratio was calculated. The kidney was then minced, and a homogenate $(10 \% \mathrm{w} / \mathrm{v})$ was prepared in chilled $1.15 \% \mathrm{KCl}$. The homogenate was used for the estimation of renal TBARS and glutathione (GSH)

The renal TBARS, an index of lipid peroxidation, were estimated according to the method described earlier. The reaction mixture was prepared by mixing $0.2 \mathrm{~mL}$ of tissue homogenate, $0.2 \mathrm{~mL}$ of $8.1 \%$ sodium dodecyl sulfate, $1.5 \mathrm{~mL}$ of $20 \%$ acetic acid solution (adjusted to $\mathrm{pH} 3.5$ with $\mathrm{NaOH}$ ), and $1.5 \mathrm{~mL}$ of $0.8 \%$ aqueous solution of TBA. The reaction mixture was made up to $4.0 \mathrm{ml}$ with distilled water and then incubated at $95^{\circ} \mathrm{C}$ for 60 -min. After cooling in tap water, $1.0 \mathrm{~mL}$ of distilled water and $5.0 \mathrm{~mL}$ of the mixture of $\mathrm{n}$-butanol and pyridine $(15: 1 \mathrm{v} / \mathrm{v})$ were added to reaction mixture and shaken vigorously using vortex shaker. The test tubes were centrifuged at 12,520 g (radius of the rotor was $7 \mathrm{~cm}$ ) for 10-min (REMI Cooling Centrifuge, India). The absorbance of the developed pink color was measured spectrophotometrically at $532 \mathrm{~nm}$. The standard curve using 1, 1, 3, 3-tertramethoxyopropane (1-10 nM) was plotted to calculate the concentration of TBARS, and the results were expressed as $\mathrm{nM} / \mathrm{g}$ wet weight of renal tissue [7].

\section{Estimation of reduced GSH}

The renal GSH level was estimated using the methods described by Ellman. Ellman's reagent (5,5'-dithiobis-(2-nitrobenzoic acid) [DTNB]) is a chemical used for measuring the amount of thiol group. Thiols react with this compound, cleaving the disulfide bond to give 2-nitro5 -thiobenzoate (NTB-), which ionizes to the NTB2- dianion in water at neutral and alkaline $\mathrm{pH}$. This NTB2-ion has a yellow color. The NTB2- is quantified in a spectrophotometer by measuring the absorbance at $412 \mathrm{~nm}$

The renal homogenate of the rat was mixed with $10 \% \mathrm{w} / \mathrm{v}$ trichloroacetic acid in $1: 1$ ratio and centrifuged at $4^{\circ} \mathrm{C}$ for 10 -min at $1957 \mathrm{~g}$. The supernatant $(0.5 \mathrm{~mL})$ was mixed with $2 \mathrm{~mL}$ of $0.3 \mathrm{M}$ disodium hydrogen phosphate buffer $(\mathrm{pH} 8.4)$ and $0.4 \mathrm{~mL}$ of distilled

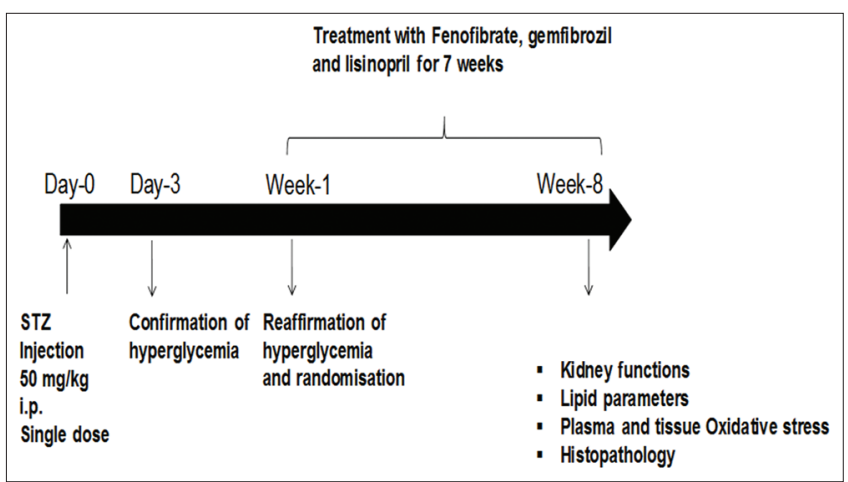

Fig. 1: Study design intervention study on diabetic nephropathy 
water. Then, $0.25 \mathrm{~mL}$ of $0.001 \mathrm{M}$ freshly prepared DTNB (dissolved in $1 \% \mathrm{w} / \mathrm{v}$ sodium citrate) was added to the reaction mixture, and then incubated for 10-min. The absorbance of the yellow colored complex was noted spectrophotometrically at $412 \mathrm{~nm}$. A standard curve was plotted using the reduced form of GSH (0.1-1 mM), and the results were expressed as $\mathrm{mM} / \mathrm{g}$ wet weight of renal tissue [7].

\section{Assessment of AKI}

The serum creatinine concentration was estimated by the alkaline picrate kinetic method using the commercially available kit (Span Diagnostics Ltd., India).

The blood urea was estimated by a method using the commercially available kit (Span Diagnostics Ltd., India).

The proteinuria was estimated by pyrogallol red method using the commercially available kit (Span Diagnostics Ltd., India) [5-7].

Histopathological study

The early diabetic changes in glomeruli were assessed histopathologically. The kidneys were excised and immediately immersed in $10 \%$ formalin. The kidney was dehydrated in graded

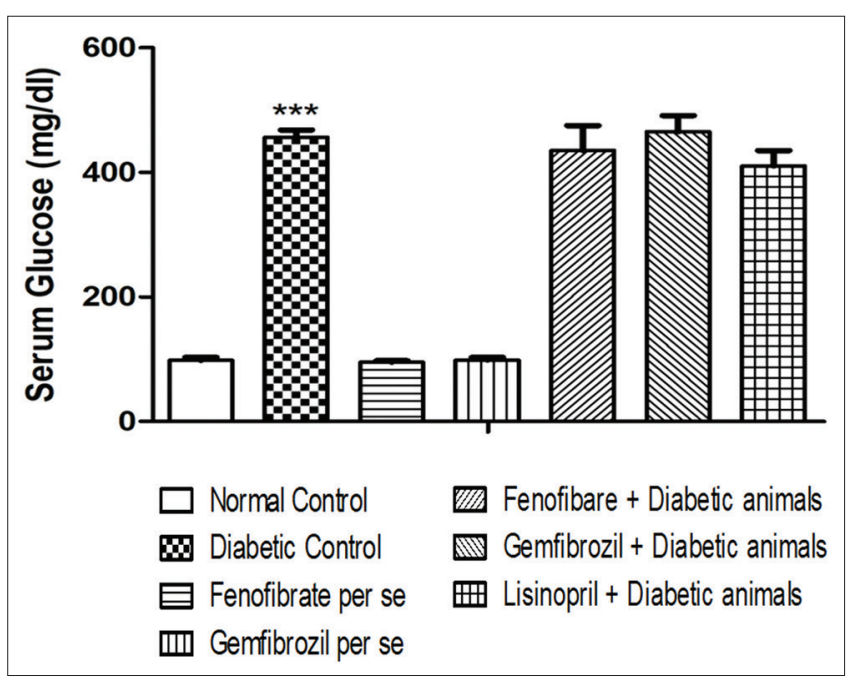

Fig. 2: Effect of fenofibrate, gemfibrozil, and lisinopril on plasma glucose in diabetic animals. All results are expressed in mean $\pm S E M .{ }^{* * *} \mathbf{p}<0.001$ versus normal control $(\mathrm{n}=6)$

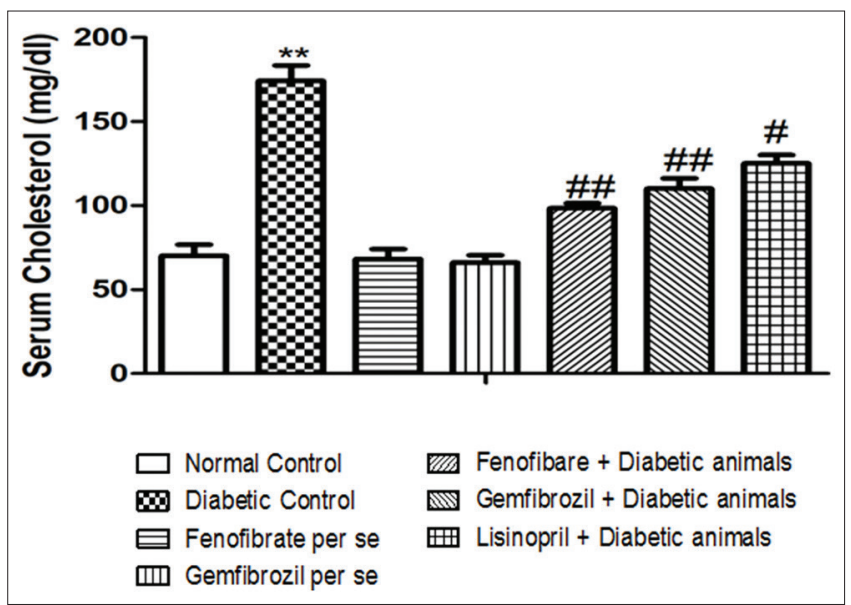

Fig. 3: Effect of fenofibrate, gemfibrozil, and lisinopril on serum cholesterol in diabetic animals. All results are expressed in mean \pm SEM. ${ }^{* *} \mathbf{p}<0.01$ versus normal control, ${ }^{\# \#} \mathbf{p}<0.01,{ }^{\#} \mathbf{p}<0.05$ versus diabetic control $(n=6)$ concentrations of alcohol, immersed in xylene and then embedded in paraffin. From the paraffin blocks, sections of $5-\mu \mathrm{m}$ thickness were made and stained with hematoxylin and eosin to assess pathological changes in glomeruli using light microscopy $(\times 400)[5,6]$.

Statistical analysis

All values were expressed as mean \pm S.E.M. The data for serum/tissue levels of TBARS, serum creatinine, blood urea and proteinuria, serum glucose, and lipid profile were statistically analyzed using one-way analysis of variance followed by Tukey's multiple comparison tests. $\mathrm{p}<0.05$ was considered to be statistically significant.

RESULT

Pharmacological account of drug in diabetic model

The administration of fenofibrate $(30 \mathrm{mg} / \mathrm{kg}$, p.o., 7 weeks) or gemfibrozil (30 mg/kg, p.o., 7 weeks) or lisinopril (1 mg/kg, p.o.,

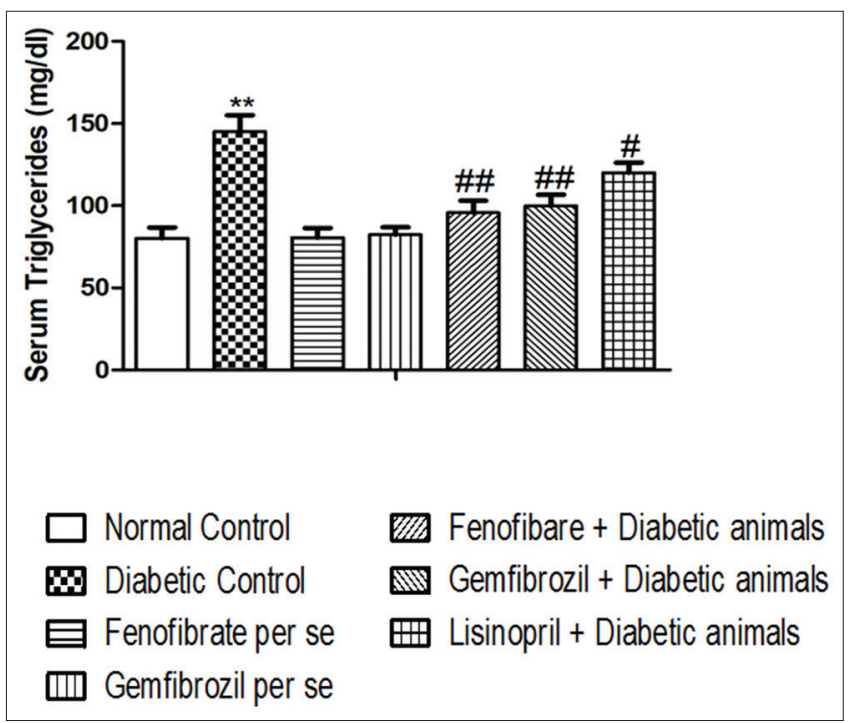

Fig. 4: Effect of fenofibrate, gemfibrozil, and lisinopril on serum triglycerides in diabetic animals. All results are expressed in mean \pm SEM. ${ }^{* *}$ p $<0.01$ versus normal control, ${ }^{\# \#} \mathbf{p}<0.01,{ }^{\#} p<0.05$ versus diabetic control $(n=6)$

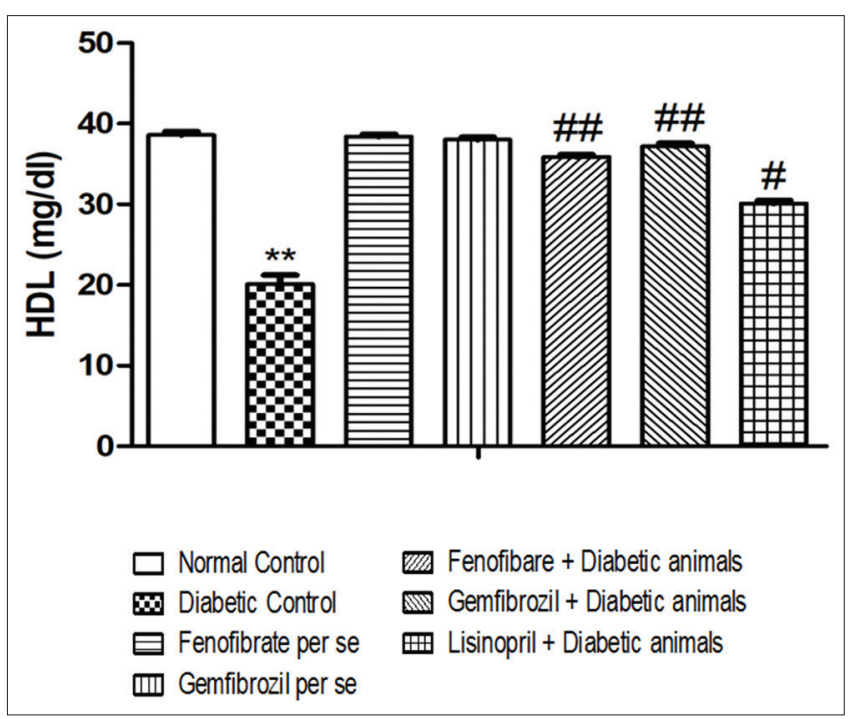

Fig. 5: Effect of fenofibrate, gemfibrozil, and lisinopril on serum high-density lipoprotein-cholesterol in diabetic animals. All results are expressed in mean $\pm S E M$. ${ }^{* *} \mathbf{p}<0.01$ versus normal control, ${ }^{\#} p<0.01, " p<0.05$ versus diabetic control $(n=6)$ 
7 weeks) to normal rats did not produce any significant per seceffects on various parameters assessed in the present study.

Administration of STZ $(50 \mathrm{mg} / \mathrm{kg}$, i.p., single dose $)$ produced hyperglycemia after $72 \mathrm{~h}$ (serum glucose $>180 \mathrm{mg} / \mathrm{dL}$ ) of injection. After 7 days of STZ administration, the rats which showed blood glucose level $>250 \mathrm{mg} / \mathrm{dl}$ were selected and were assigned in subsequent groups. Fenofibrate, gemfibrozil, and lisinopril were administered to diabetic rats after 7 days of injection of STZ, and their treatment was continued for 7 weeks. All the parameters were assessed at the end of 7 weeks of treatment, i.e., 8 weeks of diabetes induction in all groups. Overall, mortality among diabetic animals with or without drug treatments for 8 weeks was $<10 \%$.

Effect of pharmacological interventions on serum glucose

The marked increase in serum concentration of glucose was noted in diabetic rats when compared with normal rats. Treatment with fenofibrate $(30 \mathrm{mg} / \mathrm{kg}$ p.o., 7 weeks) or gemfibrozil $(30 \mathrm{mg} / \mathrm{kg}$ p.o., 7 weeks) did not affect the serum glucose concentration in diabetic rats. However, treatment with lisinopril $(1 \mathrm{mg} / \mathrm{kg}$ p.o., 7 weeks) slightly reduced the glucose level in diabetic rats; the result was not statistically significant (Fig. 2).

\section{Effect of pharmacological interventions on lipid profile}

The increase in serum concentration of total cholesterol and triglycerides and decrease in HDL were noted in diabetic rats when compared with normal rats. The treatment with fenofibrate $(30 \mathrm{mg} / \mathrm{kg}$ p.o., 7 weeks) or gemfibrozil (30 mg/kg p.o., 7 weeks) alone significantly attenuated diabetes-induced alterations in serum lipids. In addition, treatment with lisinopril partially prevented a diabetes-induced increase in total cholesterol and triglycerides and decrease in HDL level (Fig. 3-5).

\section{Effect of pharmacological interventions on serum TBARS}

The increase in serum TBARS concentration was noted in diabetic rats when compared with normal rats. However, treatment with fenofibrate (30 mg/kg p.o., 7 weeks) or gemfibrozil $(30 \mathrm{mg} / \mathrm{kg}$ p.o., 7 weeks) or lisinopril ( $1 \mathrm{mg} / \mathrm{kg}$ p.o., 7 weeks) significantly attenuated the diabetesinduced increase in serum TBARS. In addition, the marked reduction in serum TBARS was noted in diabetic rats treated with fenofibrate as compared to treatment with gemfibrozil and but not superior than lisinopril (Fig. 6).

Effect of pharmacological interventions on renal TBARS and GSH A marked increase in renal TBARS was noted in diabetic rats as compared to normal rats. In addition, a marked decrease in renal GSH was noted in diabetic rats as compared to normal rats. The treatment with fenofibrate (30 mg/kg p.o., 7 weeks) or gemfibrozil (30 mg/kg p.o., 7 weeks) or lisinopril (1 mg/kg p.o., 7 weeks) significantly attenuated the diabetesinduced increase in renal TBARS and decrease in renal GSH. In addition, the marked reduction in renal TBARS and increase in GSH was noted in diabetic rats treated with fenofibrate as compared to treatment with gemfibrozil and but not superior than lisinopril (Figs. 7 and 8).

Effect of pharmacological interventions on serum creatinine, blood urea, and proteinuria

The concentrations of serum creatinine, blood urea, and proteinuria were noted to be markedly increased in diabetic rats after 7 weeks when compared with normal rats. Treatment with fenofibrate $(30 \mathrm{mg} /$ $\mathrm{kg}$ p.o., 7 weeks) or gemfibrozil ( $30 \mathrm{mg} / \mathrm{kg}$ p.o., 7 weeks) or lisinopril ( $1 \mathrm{mg} / \mathrm{kg}$ p.o., 7 weeks) attenuated the diabetes-induced increase in serum creatinine, blood urea, and proteinuria. In addition, the marked reduction in renal serum creatinine, blood urea, and proteinuria was noted in diabetic rats treated with fenofibrate as compared to treatment with gemfibrozil and but not superior than lisinopril (Figs. 9-11).

Effect of pharmacological interventions on histopathological study on kidney

The diabetes was noted to develop pathological changes in the glomeruli such as reduced capillary size and extracellular mesangial expansion in

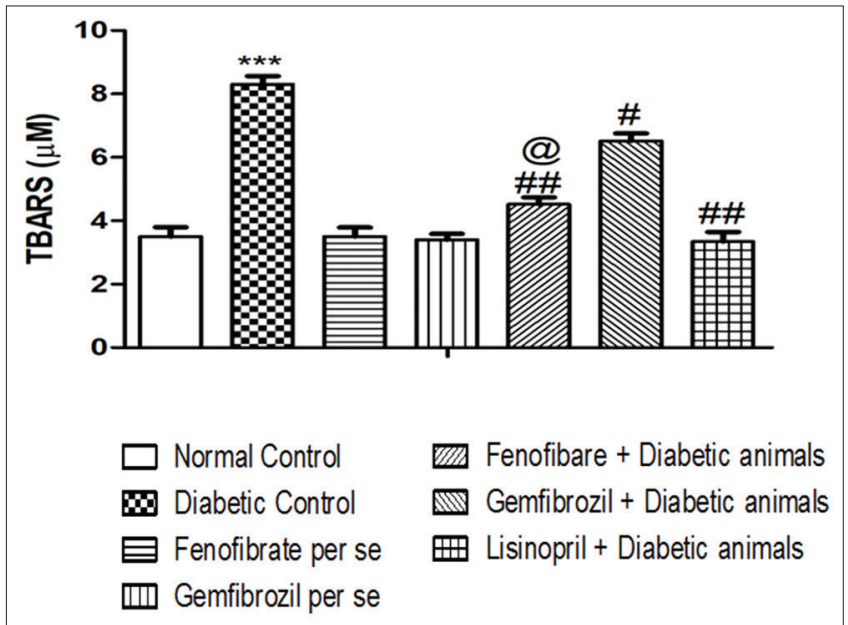

Fig. 6: Effect of fenofibrate, gemfibrozil, and lisinopril on serum TBARS in diabetic animals. All results are expressed in mean \pm SEM. ${ }^{* * *} \mathbf{p}<0.001$ vs normal control, ${ }^{\# \#} p<0.01 ; "$ p $<0.05$ vs diabetic control, ${ }^{@} \mathrm{p}<0.05$ vs gemfibrozil + diabetic animals $(\mathrm{n}=6)$

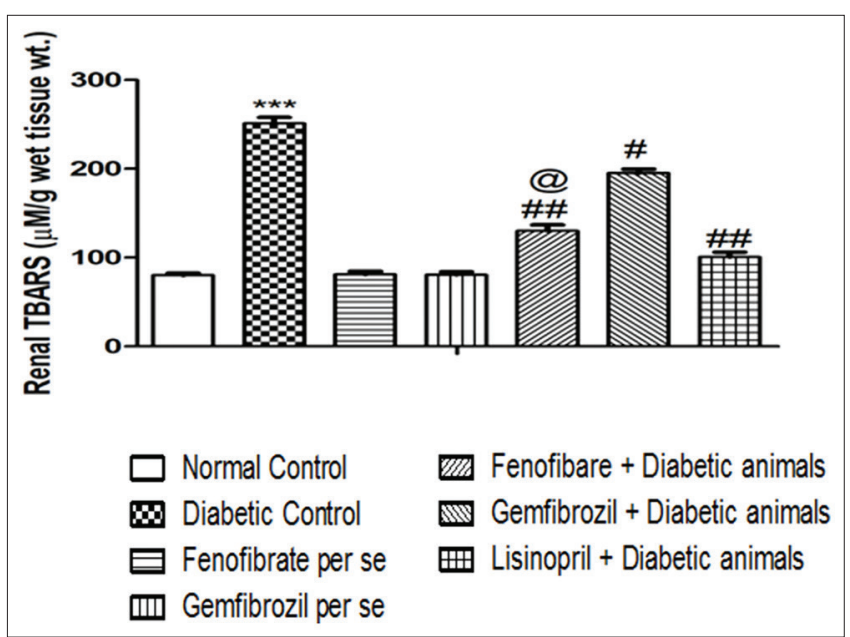

Fig. 7: Effect of fenofibrate, gemfibrozil, and lisinopril on renal thiobarbituric acid reactive substance. All results are expressed in mean \pm SEM. ${ }^{* * *} \mathbf{p}<0.001$ versus normal control, $\#$ $p<0.01$, ${ }^{\#} \mathbf{p}<0.05$ versus diabetic control, ${ }^{\oplus} \mathbf{p}<0.05$ versus gemfibrozil + diabetic animals $(n=6)$

diabetic rats after 7 weeks when compared with normal rats. Treatment with fenofibrate $(30 \mathrm{mg} / \mathrm{kg}$ p.o., 7 weeks) or gemfibrozil $(30 \mathrm{mg} / \mathrm{kg}$ p.o., 7 weeks) or lisinopril ( $1 \mathrm{mg} / \mathrm{kg}$ p.o., 7 weeks) attenuated diabetesinduced pathological changes in glomeruli. In addition, the marked pathological changes in glomeruli were noted in diabetic rats treated with fenofibrate as compared to treatment with gemfibrozil and but not superior than lisinopril (Fig. 12a-e)

\section{DISCUSSION}

One of the most important functions of the kidney is the filtration and excretion of nitrogenous waste products from the blood. The measurements of elevated blood urea nitrogen (BUN) and creatinine serve as indicators of decreased renal function indicative of the decreased clearance of these waste products. AKI is currently defined as a rapid decline in the glomerular filtration rate resulting in retention of nitrogenous wastes, primarily creatinine and BUN [8]. Concurrently, major structural hallmarks of AKI include renal enlargement, mesangial cell expansion, tubular injury, and glomerular basement membrane thickening $[9,10]$. There are several reasons for kidney damages, the 


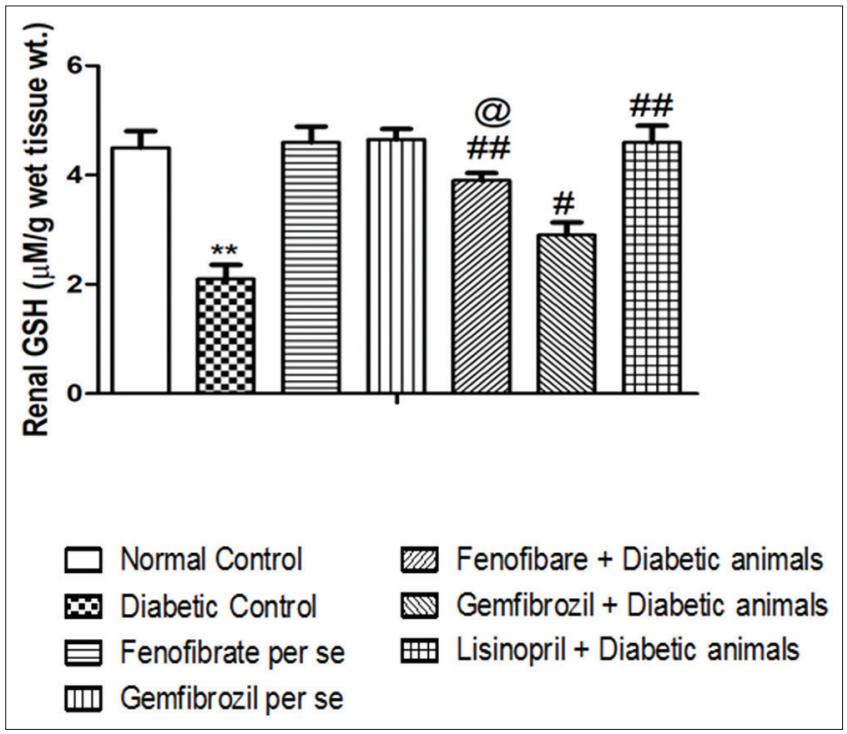

Fig. 8: Effect of fenofibrate, gemfibrozil, and lisinopril on plasma glutathione. All results are expressed in mean \pm SEM. ${ }^{* *} p<0.001$ versus normal control, ${ }^{\# \#} \mathbf{p}<0.01, "{ }^{\#}<0.05$ versus diabetic control, ${ }^{\circledR} \mathrm{p}<0.05$ versus gemfibrozil + diabetic animals $(n=6)$

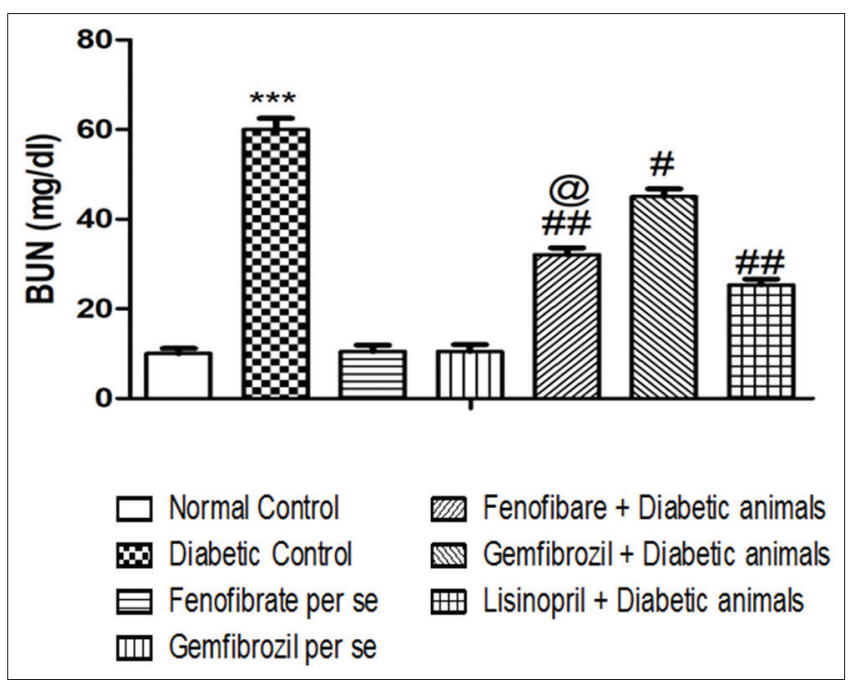

Fig. 9: Effect of fenofibrate, gemfibrozil, and lisinopril on plasma blood urea nitrogen. All results are expressed in mean \pm SEM.

${ }^{* * *} \mathbf{p}<0.001$ versus normal control, ${ }^{\# \#} \mathbf{p}<0.01,{ }^{\#} \mathbf{p}<0.05$ versus diabetic control, ${ }^{\oplus} \mathbf{p}<0.05$ versus gemfibrozil + diabetic animals $(n=6)$

primary focus on the potential causes of accounting for the majority of nephrology disease as diabetes. There are several mechanisms studied, but still, the management of kidney dysfunctions associated with diabetes remains obscure.

The animal models for nephropathy share many features which are common to human nephropathy and have been delineated by targeting proteinuria, glomerulosclerosis, glomerulonephritis, glomerular hypertrophy, tubulointerstitial nephritis, tubular necrosis, and reduced Gglomerular filtration rate. The number of patients with end-stage renal failure requiring dialysis is increasing. Therefore, suitable animal models that exhibit progressive renal lesions are necessary to identify the mechanisms involved in human nephropathy and to develop potential drugs/drug targets for the treatment of renal complications. We have used the well-established animal model of diabetes using STZ.

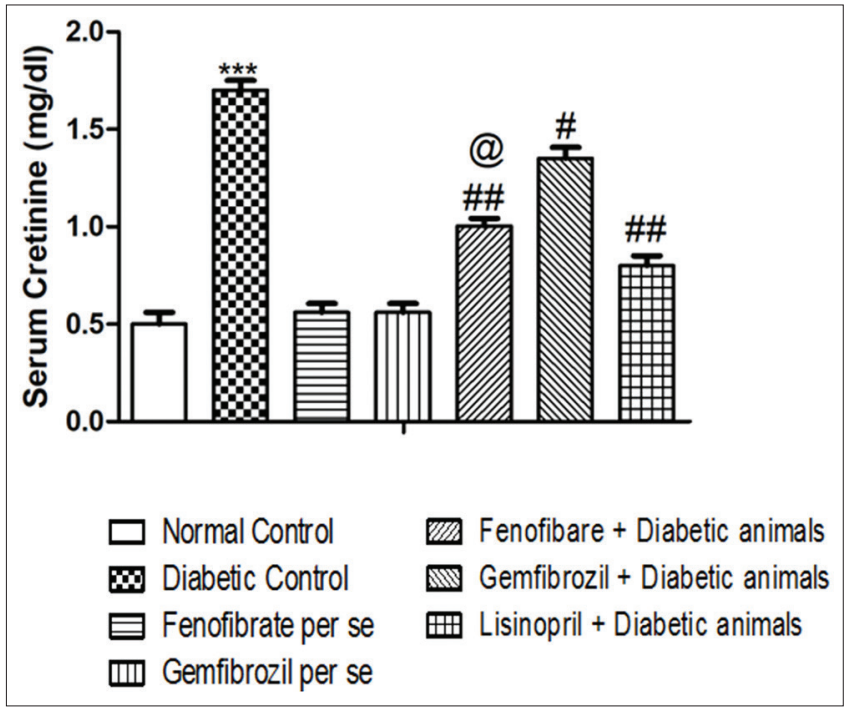

Fig. 10: Effect of fenofibrate, gemfibrozil, and lisinopril on serum creatinine. All results are expressed in mean \pm SEM. ${ }^{* *} \mathbf{p}<0.001$ versus normal control, ${ }^{\# \#} \mathbf{p}<0.01, "{ }^{\#}<0.05$ versus diabetic control, ${ }^{\circledR} \mathbf{p}<0.05$ versus gemfibrozil + diabetic animals $(n=6)$

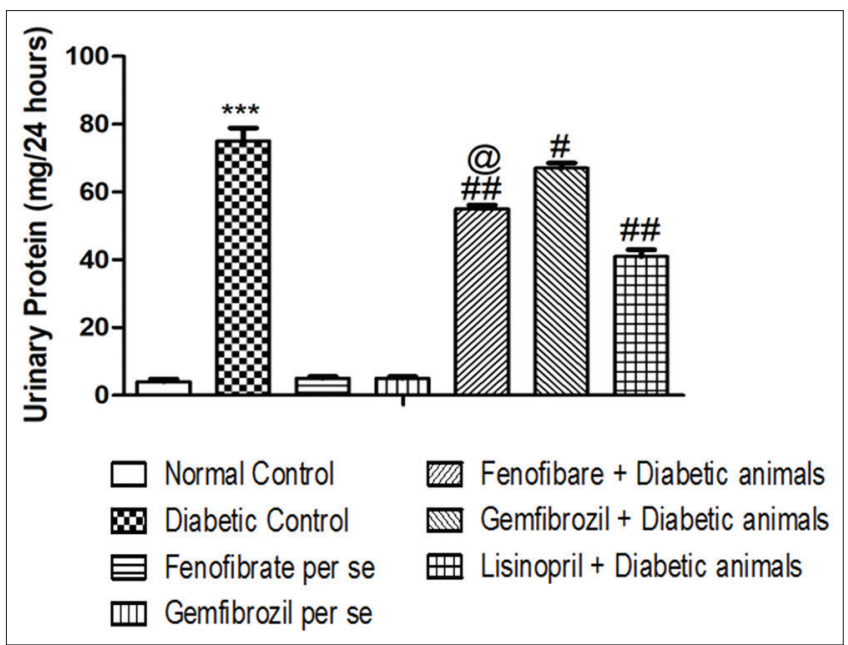

Fig. 11: Effect of fenofibrate, gemfibrozil, and lisinopril on urinary protein. All results are expressed in mean \pm SEM. ${ }^{* *} p<0.001$ versus normal control, $\# p<0.01 ; "{ }^{\#}<0.05$ versus diabetic control, ${ }^{\circledR} p<0.05$ versus gemfibrozil + diabetic animals $(n=6)$

At present, angiotensin-converting enzyme (ACE) inhibitors such as captopril, lisinopril, and fosinopril are employed to treat diabetic nephropathy [11]. However, clinical evidence suggests that ACE inhibitors are not sufficient to control the symptoms of nephropathy $[3,12,13]$. The present study has been aimed to explore the potential use of PPAR- $\alpha$ to prevent diabetic-induced AKI.

The STZ, a single dose of $50 \mathrm{mg} / \mathrm{kg}$ i.p. was employed in the present study to induce experimental diabetes in rats. STZ developed hyperglycemia within $72 \mathrm{~h}$ (serum glucose $>180 \mathrm{mg} / \mathrm{dL}$ ). After 7 days of STZ administration, rats which showed the blood glucose level $>240 \mathrm{mg} / \mathrm{dL}$ were selected for the study and are named as diabetic rats. The strong association between diabetes and vascular endothelial dysfunction and mediated nephropathy is demonstrated in various studies. Frequently, the strong correlations between oxidative stress and hyperlipidemia in the induction of nephropathy have been reported $[5,13]$. In diabetes, the reactive oxygen species (ROS) one of the main culprits plays a key role in the progression of pathophysiological processes of renal diseases. Moreover, high glucose-induced ROS generation through activation of 


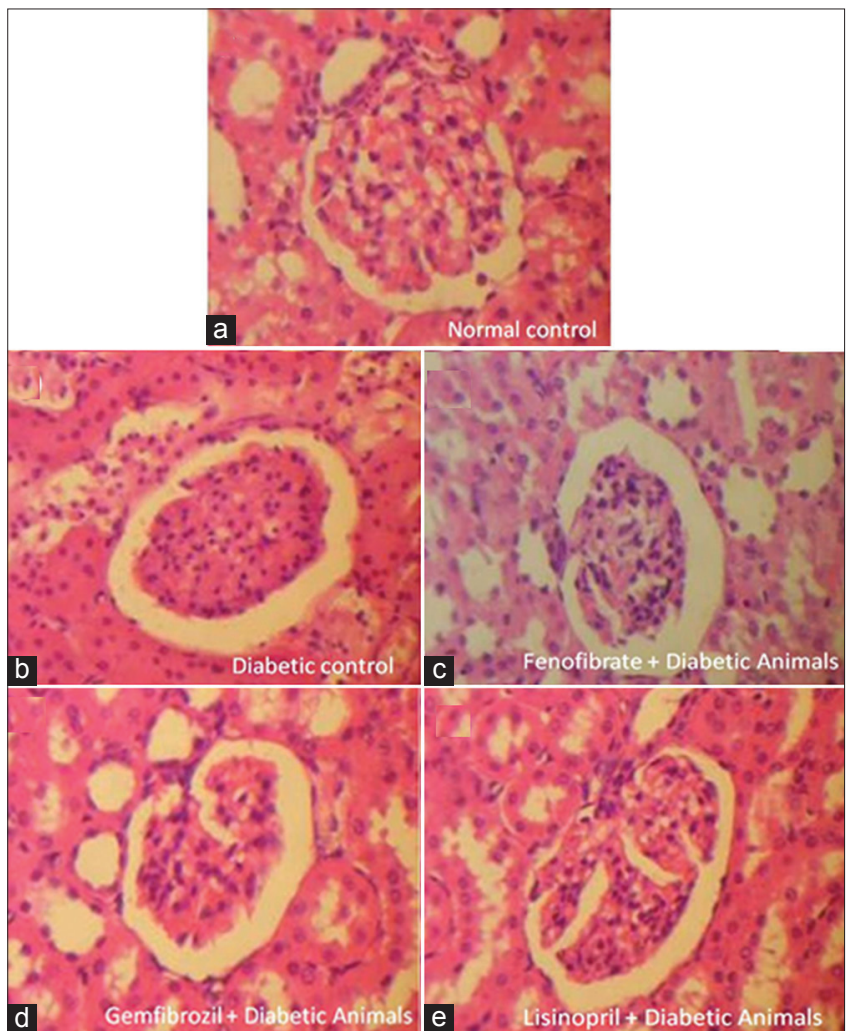

Fig. 12: (a-e) Effect of fenofibrate, gemfibrozil, and lisinopril on pathological changes in glomeruli

nicotinamide adenine dinucleotide phosphate oxidase plays a crucial role in the induction and progression of diabetic nephropathy [14]. ROS has several deleterious effects on cells, including lipid peroxidation, oxidation of cell proteins, and damage to DNA $[15,16]$. These effects can result in the loss of plasma membrane and mitochondrial membrane integrity, impaired protein function, and inhibition of cell repair and proliferation. Several studies support the fact that the oxidative stress is an important contributor to the pathogenesis of AKI. In addition, lipid peroxidation key phase is initiated by free radicals attack of membrane lipids, generating large amounts of reactive products, which have been implicated in the pathogenesis of diabetic AKI. The increase in serum and tissue TBARS level and the decrease in tissue GSH are regarded as an index of development of oxidative stress. Further, the oxidative stress has been documented to play a major role in the progression of VED and nephropathy [5]. It has been demonstrated that diabetes increases the production of ROS, lipid and consequently reduces the synthesis and bioavailability of no, which result in AKI $[5,17]$.

In the present study, diabetes has been noted to increase the renal TBARS and decrease the GSH levels in the kidney. Thus, it may be suggested that diabetes-induced development of oxidative stress may develop AKI by damaging renal architecture.

This contention is supported by the results obtained in the present study that increases in serum/tissue TBARS reduction and reduction in renal GSH and subsequently increase in serum creatinine, blood urea, and proteinuria was noted in diabetic rats when treated with interventions. However, pharmacological treatment with fenofibrate or gemfibrozil prevented diabetes-induced AKI by decreasing serum TBARS, increasing renal GSH, decreasing serum creatinine, blood urea, and proteinuria and reducing pathological changes in glomeruli.

Thus, it may be suggested that the direct renoprotective potential of fenofibrate in preventing diabetic AKI may be due to their antioxidant properties on the diabetic kidney. The overall observed beneficial effect of fenofibrate or gemfibrozil in preventing the development of diabetic
AKI may be attributed to their direct renoprotective action, improving reversing renal oxidative stress.

In diabetes, the lipid alteration besides hyperglycemia could play key roles in the development of AKI of circulating lipids could contribute to diabetic animals. Growing evidence supports the fact of significant pathologic involvement of diabetes mellitus-associated dyslipidemia in the development and progression of diabetic AKI $[5-7,18,19]$. Lipid-mediated injury plays an important role in the pathogenesis of diabetic AKI. This contention further, the increase in serum cholesterol, triglyceride, and a decrease in HDL are considered as dyslipidemia. It suggests the development of diabetes-induced hyperlipidemia. Lipoprotein lipase (LPL) located in the vascular endothelium involves the breakdown of triglycerides into free fatty acids. LPL gets downregulated during insulin deficiency [20]. The hyperlipidemia has been noted to play a role in the pathogenesis and progression of VED and nephropathy $[5,6]$.

Diabetic kidneys expressed important genes normally found in adipocytes and well suggesting a switch of kidney phenotype in favor of lipid accumulation in diabetes [21]. In diabetes, insulin inhibits the hormone-sensitive lipase production leads to abnormal high concentration of serum lipids is mainly due to increase in free fatty acids from the peripheral fat deposits and has been noted to be associated with the increased expression of transforming growth factor - $\beta 1$, fibronectin, and collagen-IV, which ultimately accounts for glomerulosclerosis and tubulointerstitial fibrosis $[19,20]$. This contention is supported by the results obtained in the present study that marked increases in serum cholesterol and triglycerides and consequent decrease in serum HDL levels were noted in diabetic rats with AKI. Decreased expression of PPAR- $\alpha$ has been noted to be associated with the progression of diabetic nephropathy through an increase in circulating lipids, induction of inflammation, and extracellular matrix formation in diabetic mice [22]. In the present study, the increase in serum concentration of total cholesterol and triglycerides and a decrease in HDL and increase in serum concentration were noted in both diabetic rats when compared with normal rats, which indicates both diabetes induced-hyperlipidemia. Similar kind of lipid abnormality has been noted by previous reports on the same model $[23,24]$.

The diabetes-induced alteration in lipid profile such as hypercholesterolemia and hypertriglyceridemia affect the function of the glomerulus and produce glomerulosclerosis, which results in diabetic nephropathy [24]. Further, insulin has an inhibitory action on 3-hydroxy-3-methyl-glutaryl-Co-A (HMG-COA) reductase, a key rate-limiting enzyme involved in the synthesis of cholesterol. Hence, hypoinsulinemia during diabetes activates HMG-COA reductase to stimulate the synthesis of high cholesterol [25]. Moreover, the occurrence of proteinuria has been suggested to upregulate HMG-COA reductase to produce hypercholesterolemia [26,27]. Accordingly, it may be suggested that diabetes-induced hyperlipidemia may develop AKI by damaging renal architecture. In the present study, the serum creatinine, blood urea, and proteinuria were noted to be increased in diabetic rats as compared to normal rats. Moreover, diabetic rats developed pathological changes in glomeruli in 7 weeks. These results suggest the development of diabetes-induced AKI.

The biochemical alteration and morphological changes in the kidney were considered as a primary marker for the induction of AKI. The STZ (single dose) used for the induction of diabetes in rats. The renoprotective effect of lisinopril has been well reported in basic and clinical studies $[5,28]$. Therefore, lisinopril has been employed as a standard drug in the present study. Renoprotection afforded by lisinopril might be, in some measure, its glucose-lowering diabetic rats. As dyslipidemia is common consequences of renal dysfunction, PPAR- $\alpha$ agonists could serve as promising therapeutic agents for controlling the progression of diabetic nephropathy. In fact, fenofibrate, a hypolipidemic agent acts as a PPAR- $\alpha$ agonist, reduced renal lipotoxicity, inflammation, fibrosis, and subsequently prevented the symptoms of diabetic AKI. Further, 
gemfibrozil the other PPAR- $\alpha$ agonist noted to be useful to control diabetic dyslipidemia and control diabetic nephropathy. Importantly, the fenofibrate was noted to be superior in preventing diabetic AKI than gemfibrozil. The present work emphasized the rationale for employing PPAR- $\alpha$ agonists in the management of diabetic nephropathy.

\section{Structural abstract}

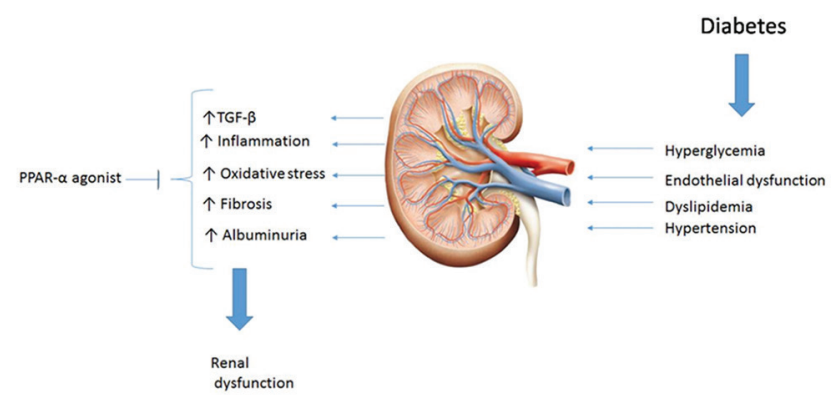

\section{CONCLUSION}

On the basis of above discussion, diabetes-mediated lipid alteration and oxidative stress could play a role in the development of AKI. Fenofibrate and gemfibrozil show antihyperlipidemic, antioxidant property, and contributed in renoprotection in diabetic rats with AKI. Apart from antihyperlipidemic action fenofibrate showed, the significant renoprotection action of than gemfibrozil by drastically restructures the kidney architect.

\section{ACKNOWLEDGMENT}

I express my deep sense of gratitude to my esteemed teacher and guideDr. Pravin S. Kawtikwar, Principal, Sudhakarrao Naik Institute of Pharmacy, Pusad Maharashtra. His inspiring and priceless guidance and his enthusiasm and a never-ending drive for work were a constant source of inspiration for me.

\section{AUTHOR'S CONTRIBUTION}

VA Chakkarwar, research scholar, constructing an idea or hypothesis for research and/or manuscript. Planning methodology to reach a conclusion. Reviewing the article before submission not only for spelling and grammar but also for its intellectual content. P.S. Kawtikwar, supervising, organizing, and supervising the course of the project or the article along with constructing a hypothesis and taking the responsibility to complete research work.

\section{CONFLICTS OF INTEREST}

None.

\section{REFERENCES}

1. Rossing P, de Zeeuw D. Need for better diabetes treatment for improved renal outcome. Kidney Int Suppl 2011;120:S28-32.

2. Star RA. Treatment of acute renal failure. Kidney Int 1998;54:1817-31.

3. Sharma D, Bhattacharya P, Kalia K, Tiwari V. Diabetic nephropathy: New insights into established therapeutic paradigms and novel molecular targets. Diabetes Res Clin Pract 2017;128:91-108.

4. Sharina IG, Sobolevsky M, Papakyriakou A, Rukoyatkina N, Spyroulias GA, Gambaryan S, et al. The fibrate gemfibrozil is a NO- and haem-independent activator of soluble guanylyl cyclase: In vitro studies. Br J Pharmacol 2015;172:2316-29.

5. Balakumar P, Chakkarwar VA, Singh M. Ameliorative effect of combination of benfotiamine and fenofibrate in diabetes-induced vascular endothelial dysfunction and nephropathy in the rat. Mol Cell
Biochem 2009;320:149-62.

6. Balakumar P, Varatharajan R, Nyo YH, Renushia R, Raaginey D, Oh AN, et al. Fenofibrate and dipyridamole treatments in low-doses either alone or in combination blunted the development of nephropathy in diabetic rats. Pharmacol Res 2014;90:36-47.

7. Kadian S, Mahadevan N, Balakumar P. Differential effects of low-dose fenofibrate treatment in diabetic rats with early onset nephropathy and established nephropathy. Eur J Pharmacol 2013;698:388-96.

8. Dhodi JB, Mestry SN, Juvekar AR. Diabetic nephropathy-genesis, prevention, and treatment. Int J Pharm Pharm Sci 2014;6:42-7.

9. Hu C, Sun L, Xiao L, Han Y, Fu X, Xiong X, et al. Insights into the mechanisms involved in the expression and regulation of extracellular matrix proteins in diabetic nephropathy. Curr Med Chem 2015;22:2858-70.

10. Fioretto P, Mauer M. Histopathology of diabetic nephropathy. Semin Nephrol 2007;27:195-207.

11. Senthilkumar N, Anandhasayanam A, Safil KM, Shemimol S. A comparative study on the effectiveness of angiotensin converting enzyme inhibitors (ACEIS) and angiotensin receptor blockers (ARBS) in diabetic nephropathy in Type 2 diabetes mellitus patients. Int J Pharm Pharm Sci 2016;8:289-92.

12. Zhao HJ, Wang $\mathrm{S}$, Cheng $\mathrm{H}$, Zhang MZ, Takahashi T, Fogo AB, et al. Endothelial nitric oxide synthase deficiency produces accelerated nephropathy in diabetic mice. J Am Soc Nephrol 2006;17:2664-9.

13. Hayashi D, Ueda S, Yamanoue M, Ashida H, Shirai Y. Amelioration of diabetic nephropathy by oral administration of $\mathrm{d}-\alpha$-tocopherol and its mechanisms. Biosci Biotechnol Biochem 2018;82:65-73.

14. Arora MK, Singh UK. Molecular mechanisms in the pathogenesis of diabetic nephropathy: An update. Vascul Pharmacol 2013;58:259-71.

15. Palm F. Intrarenal oxygen in diabetes and a possible link to diabetic nephropathy. Clin Exp Pharmacol Physiol 2006;33:997-1001.

16. Griendling KK, Minieri CA, Ollerenshaw JD, Alexander RW. Angiotensin II stimulates NADH and NADPH oxidase activity in cultured vascular smooth muscle cells. Circ Res 1994;74:1141-8.

17. Balakumar P, Chakkarwar VA, Krishan P, Singh M. Vascular endothelial dysfunction: A tug of war in diabetic nephropathy? Biomed Pharmacother 2009;63:171-9.

18. Wolf G, Ziyadeh FN. Cellular and molecular mechanisms of proteinuria in diabetic nephropathy. Nephron Physiol 2007;106:26-31.

19. Taskinen MR. 3 hyperlipidaemia in diabetes. Baillieres Clin Endocrinol Metab 1990;4:743-75.

20. Figarola JL, Loera S, Weng Y, Shanmugam N, Natarajan R, Rahbar S, et al. LR-90 prevents dyslipidaemia and diabetic nephropathy in the zucker diabetic fatty rat. Diabetologia 2008;51:882-91.

21. Allain CC, Poon LS, Chan CS, Richmond W, Fu PC. Enzymatic determination of total serum cholesterol. Clin Chem 1974;20:470-5.

22. Park CW, Kim HW, Ko SH, Chung HW, Lim SW, Yang CW, et al. Accelerated diabetic nephropathy in mice lacking the peroxisome proliferator-activated receptor alpha. Diabetes 2006;55:885-93.

23. Ali MM, Agha FG. Amelioration of streptozotocin-induced diabetes mellitus, oxidative stress and dyslipidemia in rats by tomato extract lycopene. Scand J Clin Lab Invest 2009;69:371-9.

24. Kang HW, Lim WC, Lee JK, Ho JN, Lim EJ, Cho HY, et al. Germinated waxy black rice ameliorates hyperglycemia and dyslipidemia in streptozotocin-induced diabetic rats. Biol Pharm Bull 2017;40:1846-55.

25. Sevak AR, Goyal RK. Effects of chronic treatment with lisinopril on cardiovascular complications in streptozotocin diabetic and DOCA hypertensive rats. Pharmacol Res 1996;34:201-9.

26. Trevisan R, Dodesini AR, Lepore G. Lipids and renal disease. J Am Soc Nephrol 2006;17 4 suppl 2:S145-7.

27. Vaziri ND, Sato T, Liang K. Molecular mechanism of altered cholesterol metabolism in focal glomerulosclerosis. Kidney Int 2003;63:1756-63.

28. Amann B, Tinzmann R, Angelkort B. ACE inhibitors improve diabetic nephropathy through suppression of renal MCP-1. Diabetes Care $2003 ; 26: 2421-5$. 